\title{
Brain auditory evoked response test, the standard method for the diagnosis of the hereditary deafness in dogs
}

\author{
Nicolae Coldea ${ }^{1, *}$ \\ 1 "Dr Coldea Nicolea" private practice, 1C Tudor Arghezi St., Sibiu, Romania: coldeadvm@yahoo.com \\ * Correspondence: coldeadvm@yahoo.com;
}

\begin{abstract}
Hearing deficiency is one of the most common hearing impairments that affect humans and other mammalian alike. Hearing loss is not painful or a life-threatening change but can endanger the patient by taking into account a large number of breeds predisposed to hereditary deafness, this short communication aims to synthesize the steps and the method for BAER test. For the affected breeds, the BAER test is recommended starting at the age of two months.
\end{abstract}

Keywords: BAER, dog, auditory evoked response test

Received: 08.05.2021

Accepted: 03.09.2021

Published: 29.092021

DOI:10.52331/cvj.v26i2.23

Copyright: () 2021 by the authors. Submitted for possible open access publication under the terms and conditions of the Creative Commons Attribution (CC BY) license (http://creativecommons.org/licenses /by/4.0/).

\section{Introduction}

Dog breeds that are affected by hereditary deafness: Akita, Dalmatian, Nova Scotia, Duck Tolling Retriever, Alapaha Blue Blood Bulldog/Otto Bulldog, Dappled Dachshund, Old English Sheepdog, American Bulldog, Doberman Pinscher, Papillon, American-Canadian Shepherd, Dogo Argentino, Pekingese, American Eskimo, English Bulldog, Perro de Carea Leones, American Hairless Terrier, English Cocker Spaniel, Pit Bull Terrier, American Staffordshire Terrier, English Setter Pointer/English Pointer, Anatolian Shepherd, Foxhound, Presa Canario, Australian Cattle Dog, Fox Terrier, Puli, Australian Kelpie, French Bulldog, Rhodesian Ridgeback, Australian Shepherd, German Shepherd, Rat Terrier, Australian Stumpy-tail Cattle Dog, German Shorthaired Pointer, Rottweiler, Beagle, Goldendoodle, Saint Bernard, Belgian Sheepdog/Groenendael, Great Dane, Saluki, Belgian Tervuren, Great Pyrenees, Samoyed, Bichon Frise, Greater Swiss Mountain Dog, Schnauzer Border Collie Greyhound Scottish Terrier Borzoi Havanese Sealyham Terrier Boston Terrier, Ibizan Hound, Shetland Sheepdog, Boxer, Icelandic Sheepdog, Shih Tzu, Brittney Spaniel, Italian Greyhound, Shropshire Terrier, Bulldog Jack/Parson Russell Terrier, Siberian Husky, Bullmastiff, Japanese Chin, Soft Coated Wheaten Terrier, Bull Terrier, Keeshond, Springer Spaniel, Canaan Dog, Kuvasz, Sussex Spaniel, Cardigan Welsh Corgi, Labrador Retriever, Tibetan Spaniel, Catahoula Leopard Dog, Lhasa Apso, Tibetan TerrierCatalan Shepherd LowchenToy Fox Terrier Cavalier King Charles Spaniel Maltese, Toy Poodle, Chihuahua, Manchester Terrier, Walker American Foxhound, Chinese Crested Miniature Pinscher, West Highland White Terrier, Chow Chow, Miniature Poodle, Whippet, Cocker Spanielmongrel, Yorkshire Terrier, Collie, Newfoundland Landseer, Coton de Tulear, Norwegian Dunkerhound [1].

For these dogs, the BAER test is recommended starting at the age of two months.

This paper aims to describe the technique of the test and the interpretation of the results. We will not insist on the etiology of deafness as it is described elsewhere $[2,1,3]$. However, some elements of classification, anatomy, physiology, and genetics are necessary. 


\section{Deafness}

Deafness can be peripheral or central, congenital / inherited or acquired, uni- or bilateral, total or partial, conductive or neurosenzorial.

The congenital deafness should not be confused with the hereditary one; the congenital form may appear during parturition and is determined by dystocia and oxygen deprivation. Hereditary deafness occurs 3 to 4 weeks after birth and is a neurosensorial type. It is the result of the degeneration of a structure called stria vascularis. This structure is responsible for maintaining a very specific composition of the endolymph from scala media, characterized by a high concentration of potassium ions and a low concentration of sodium ions, different from other extracellular fluids inside the body. This process is involved the numerous melanocytes present in stria vascularis[3,4].

When the atrophy of the stria occurs and/or the melanocytes are missing, the composition of the endolymph is altered and leads to the death of the hair cells from the organ of Corti. The hair cells are the ones who transformed the sound (mechanic oscillations) transmitted to the cilia by the tectorial membrane in electric impulses. When these structures are damaged, peripheral neurosenzorial deafness appears.

A different situation is described in Dobermans when the hair cells die, but the melanocytes from stria vascularis are intact, called neuroepithelial deafness [5,6].

The inheritance of deafness is controlled by a gene situated on Merle (M) locus, with two alleles: the recessive $\mathrm{m}$ and the dominant $\mathrm{M}$. The homozygous $\mathrm{MM}$ individuals present blue irises and may be blind and sterile. Unfortunately, heterozygous Mm x Mm parents will give birth to 25\% MM puppies.

Another gene is placed on the $S$ locus, and it is called piebald. It has four alleles: dominant (S) and recessive $\left(\mathrm{s}^{\mathrm{i}}\right)$ Irish spotting, (sp) piebald, and (SW) extreme white piebald. The inheritance mechanism is considered to best fit by the presence of two autosomal recessive genes or an incompletely penetrant recessive allele.

Regarding the deafness in Dobermans, the inheritance has an autosomal recessive mechanism [3].

\section{BAER test}

According to the "Orthopedic Foundation for Animals," the only accepted test (at least for the moment) to detect hereditary deafness in dogs is the BAER (Brain Auditory Evoked Response) test.

Headphones deliver the test sound and one ear at a time test can be diagnostic for other types of acquired deafness (toxic, traumatic, degenerative, inflammatory, etc.) (fig. 1). It is recommended to perform the test at the age of two months. The test shows a few waves, and the first five ones are of diagnostic importance. The waves are marked with roman numbers (fig. 1).

Wave I is generated by the distal end of the cranial nerve VIII, wave II by the proximal part of cranial nerve VIII, wave III by the cochlear nucleus, wave IV and V inferior colliculus, and probably by the medial geniculate body. Anyway, the disturbances responsible for altering the last two waves will determine other symptoms of diseases of the central nervous system.

The test can be done without sedation, but if the patient is restless, it can be sedated because the medication does not affect the test result. Currently, we are using four electrodes: one "grounding" electrode at the level of the T1 vertebra, one positive electrode on the median line of the skull at the level of the ears, and two negative electrodes anterior to the tragus of each ear. 


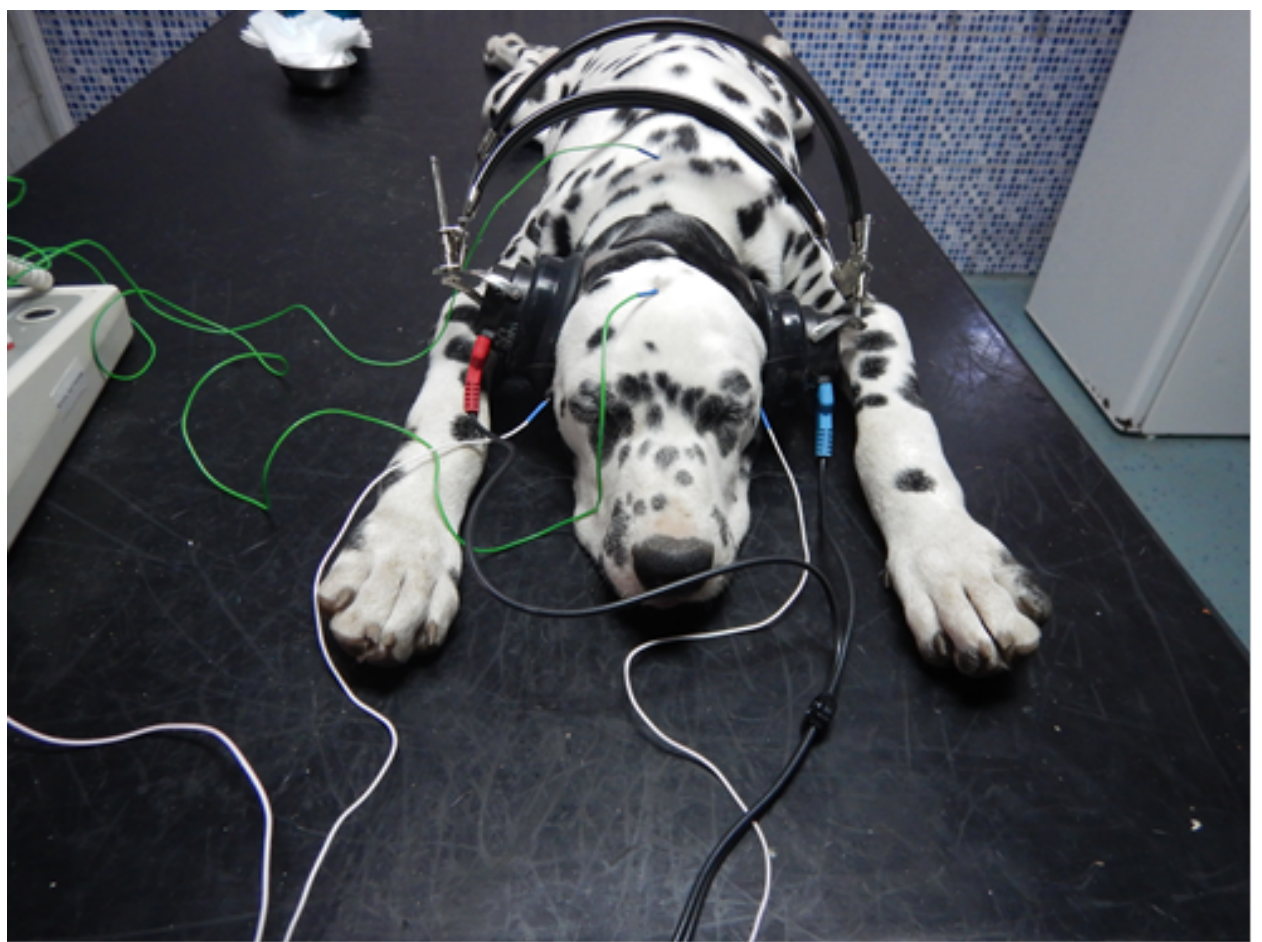

Fig. 1 Positioning of headphones in BAER test
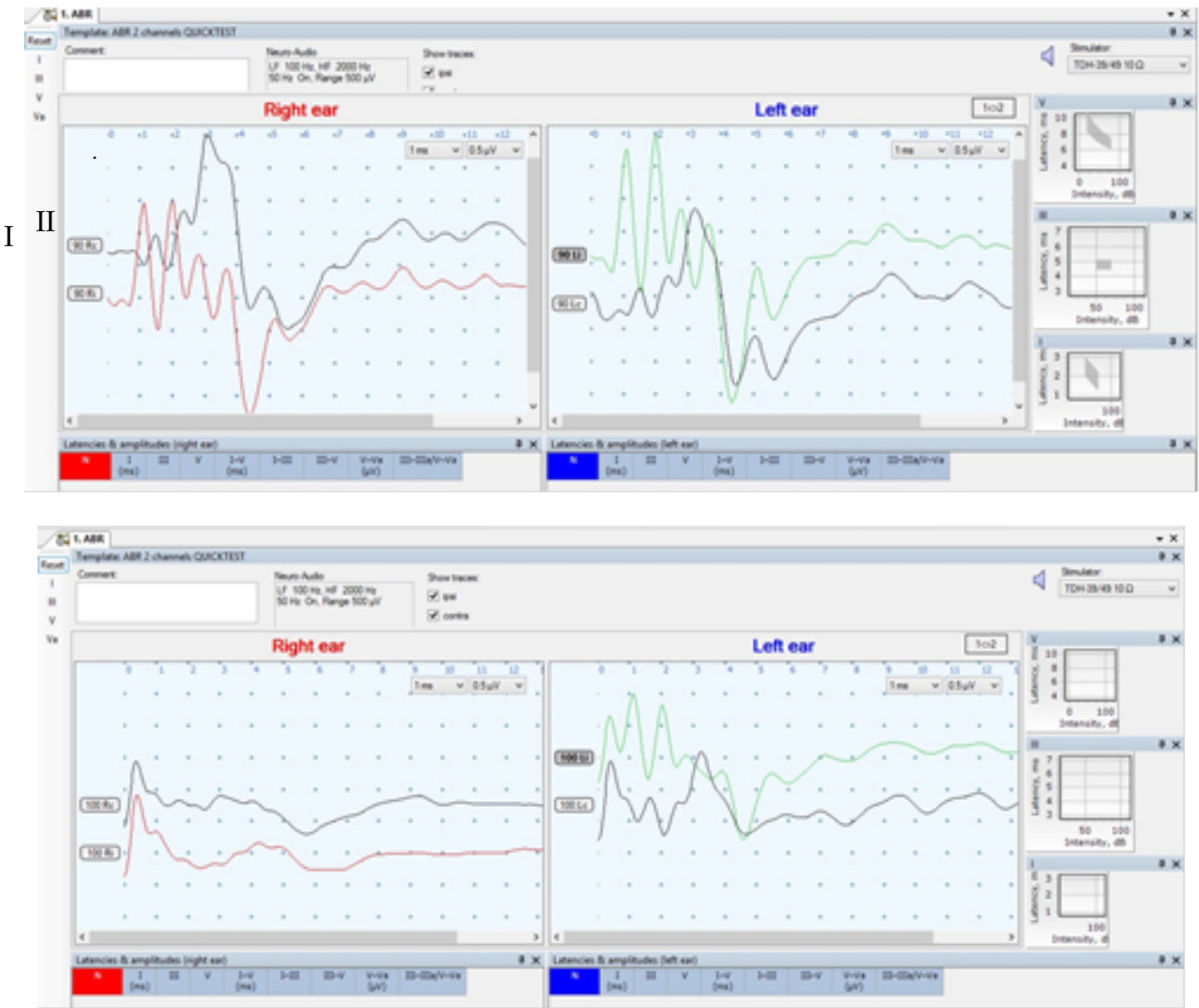

Fig. 2 Right ear deafness. 


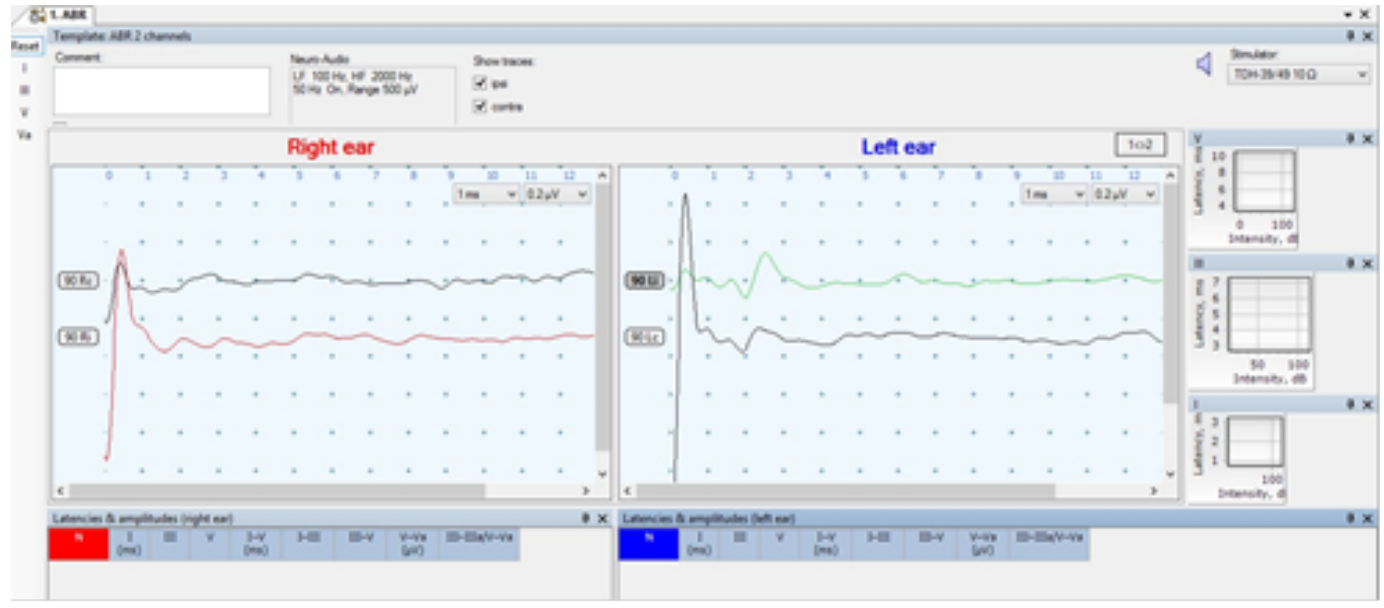

Fig. 3 Bilateral deafness.

To avoid motion artifacts, sedation of the patient is recommended.

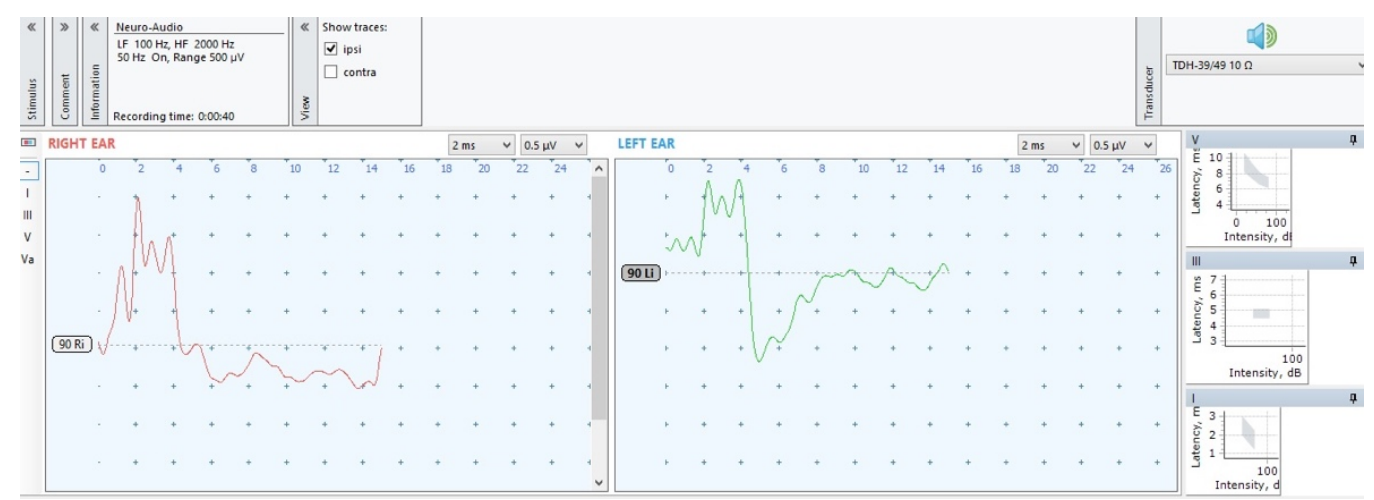

Fig. 4 Motion artifact

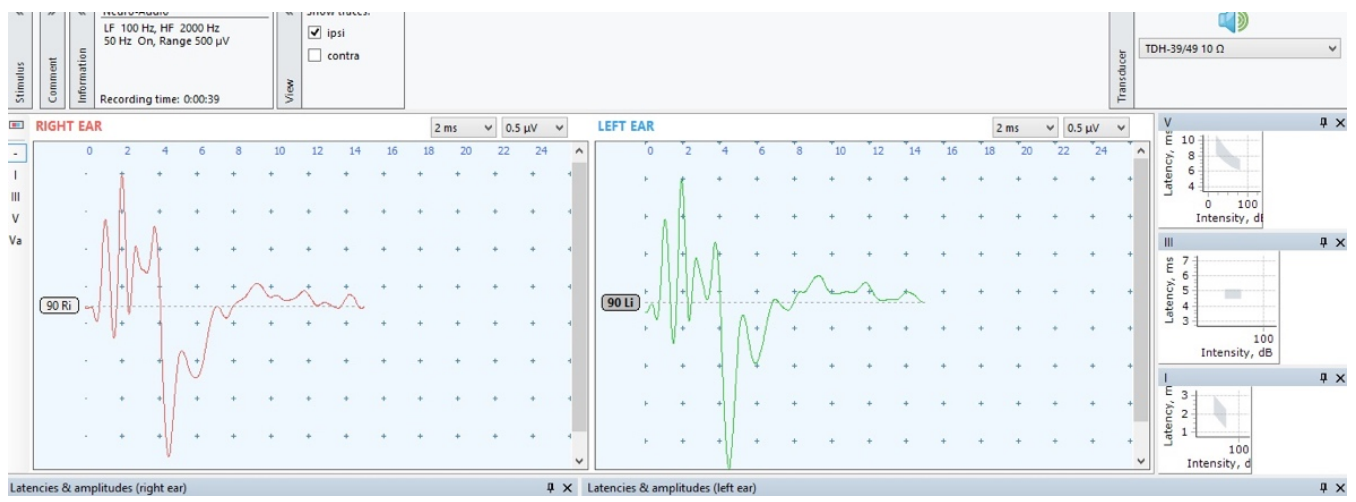

Fig. 5. Same patient after sedation.

\section{Discussion}

Identification of the dogs (e.g., microchip) is very important because uni- or bilateral deaf dogs must be excluded from breeding. Puppies with unilateral deafness can be excellent pets, but those with bilateral deafness need special attention. These dogs can be good companion animals, but care should be taken to avoid unattended contact with other persons or animals. Because they cannot hear, they may have a violent reaction when touched without warning. Anytime someone wants to pet the dog, he should be sure that he is within the visual range of the affected dog. It is recommended to provide these patients with collars or harnesses with warnings (e.g., Don't touch! Deaf dog!) One can find/buy this type of equipment in pet shops or on the internet. It is highly recommended to use them, to avoid accidents. 


\section{Conflicts of Interest: None}

\section{References}

1. Strain, G.M. Canine Deafness. Veterinary Clinics of North America: Small Animal Practice 2012, 42, 1209-1224, doi: https://doi.org/10.1016/j.cvsm.2012.08.010

2. Strain, G.M. Aetiology, Prevalence and Diagnosis of Deafness in Dogs and Cats. British Veterinary Journal 1996, 152, 17-36, doi: https://doi.org/10.1016/S0007-1935(96)80083-2.

3. Strain, G.M. The Genetics of Deafness in Domestic Animals. Front. Vet. Sci. 2015, 2, doi: https://doi.org/10.3389/fvets.2015.00029.

4. Holliday, T.A.; Nelson, H.J.; Williams, D.C.; Willits, N. Unilateral and Bilateral Brainstem Auditory-Evoked Response Abnormalities in 900 Dalmatian Dogs. Journal of Veterinary Internal Medicine 1992, 6, 166-174, doi: https://doi.org/10.1111/j.19391676.1992.tb00332.x.

5. Wilson, W.J.; Mills, P.C. Brainstem Auditory-Evoked Response in Dogs. American Journal of Veterinary Research 2005, 66, 21772187, doi: https://doi.org/10.2460/ajvr.2005.66.2177.

6. Wilson, W.J.; Mills, P.C.; Bradley, A.P.; Petoe, M.A.; Smith, A.W.B.; Dzulkarnain, A.A. Fast Assessment of Canine Hearing Using High Click-Rate BAER. The Veterinary Journal 2011, 187, 136-138, doi: https://doi.org/10.1016/j.tvjl.2009.10.009. 